\title{
Treatment of chronic osteomyelitis in children by llizarov technique
}

\begin{abstract}
Chronic osteomyelitis in children is very difficult to eradicate completely. The patient with chronic osteomyelitis has a new hope of cure by recent advances in the management of chronic osteomyelitis. Systemic manifestations may subside, but one or more foci in the bone may contain purulent material, infected granulation tissue or a sequestrum. Treatment of this condition is to irradiate the infection and to restore the functional activity of the patient. Our objective is to highlight the various causes of recurrence, relevant classification, pathophysiology and modern treatment of chronic osteomyelitis with Ilizarov technique. ${ }^{1-3}$
\end{abstract}

Keywords: Chronic osteomyelitis, Radical resection, Ilizarov
Volume 2 Issue 4 - 2015

\author{
Bari MM, ${ }^{1,2}$ Shahidul Islam,' Mahfuzer \\ Rahman,' Shetu NH,' Golam Mostafa,' \\ Naima Ferdousi' \\ IDepartment of Orthopaedics, Bari-llizarov Orthopaedic \\ Centre, Bangladesh \\ 2Visiting and Honored Prof., Russian Ilizarov Scientific Centre, \\ Russia
}

Correspondence: Mofakhkharul Bari, Bari-llizarov Orthopaedic Centre, 72, Satmasjid Road, Nizams Shankar Plaza, Dhanmondi, Dhaka, Bangladesh, Tel +880I819211595, Email bari.ilizarov3I@gmail.com

Received: April 12, 2015 | Published: April 18, 2015

\section{Introduction}

Recent advances in the management of chronic osteomyelitis in children includes

a. Radical resection and bone transport. ${ }^{4-6}$

b. Improving nutritional status. ${ }^{7}$

c. Anatomical and physiological emphasis on classification (CiernyMader $)^{6}$

d. Newer antibiotics. ${ }^{8}$

\section{Chronic osteomyelitis is often associated with}

i. angular or rotational deformity, the angle may be in any plane such as procurvatum, recurvatum, varus, valgus or in the oblique plane

ii. Deformities of the adjacent joints.

iii. Limb length discrepancy (LLD)

iv. A deep cavity in the bone

v. A big sequestum, which creates a big gap.

\section{What are the causes of chronic osteomyelitis?}

a. Academician Professor llizarov [9] said, in chronic osteomyelitis the tiny cavities are filled with infective organisms around the large fixed focus. The small cavities are the causes of recurrence. With llizarov fixator; if you want you can fill this gap by bone transport. This gap can be filled by bone transport. The very big advantage of llizarov method is that one can simultaneously correct all the deformities and we can get excellent stability by inserting bio-compatible thin wires and securing it with the rings.

b. Decreased blood flow as a result of initial insult or operative procedure diminishes the healing capacity. Another factor is that the thrombosis of the blood vessel surrounding the infected area also reduces the blood supply. c. Antibiotics cannot penetrate though the infected and necrotic area and sequestrum produces an area of lowered vascularity.

d. Resistance of organisms to antibiotics. Organism forms a biofilm around the sequestrum or implant.

e. Inadequate surgical debridement.

In children sometimes you don't need to perform radical resection, even if you find pandiaphyseal osteomyelitis with pathological fracture, and persistent discharging sinus. It is a waste of money to advice intravenous antibiotic for a long time.

\section{Anatomic classification or adult chronic osteomyelitis is of 4 types}
A. Intramedullary
B. Superficial
C. Local and
D. Diffuse.

\section{Materials and methods}

\section{Study places}

i. NITOR (National Institue of Traumatology and Orthopaedic Rehabilitation), Dhaka.

ii. BIO (Bari-llizarov Orthopaedic) Centre, Dhanmondi, Dhaka.

iii. Mymensingh Medical College and Hospital.

iv. Narayangong 200 Bedded Hospital.

Duration: January 1993 to January 2013

Number of Children: 165 ( 85 were severe)

Age range: 3-15 years

Follow-up period: 2-19 years. 


\section{The cases were treated as follows}

a. Thorough debridement; removal of sequestri, curreting cavity.

b. Acute docking, if acute docking is not possible, then gradual docking is done.

c. Condrodiastasis or corticotomy and application of llizarov frame. Gaps were closed by bone transport. Lengthening was done as required. Deformities were corrected by the llizarov frame.

d. Pan-diaphyseal osteomyelitis with pathological fractures were treated by only the stable fixation of llizarov with full weight, bearing and motion'.

In adult we must identify the organisms. Clinical staging can be done according to Cierny-Mader classification. This classification has developed an anatomical situation of chronic osteomyelitis and physiologic response of the host are taken into consideration.

\section{Radical resections}

The treatment of chronic osteomyelitis should be similar to treatment of giant cell tumor of bone. Surgical debridement is the main th9ing of treatment in chronic osteomyelitis

1. All the infected tissues, sequestri dead avascular bone are removed.

2. Implants must be removed.

3. The cavity is cleaned with jet average. The bony ends are debrided until the punctate bleeding is seen (Paprika Sign + ).

4. Severely infected and sclerosed bony segment is removed. The gap created can be filled by bone transport.

Noramal saline is used for cleaning and when oozing Haversian systems are visualized, we stop here.

\section{Treatment of cavity}

With the liizarov method a well off bone above or below the cavity can be transported across the cavity which can be completely closed.

\section{Bone gap and bone transport}

In chronic osteomyelitis: Academician professor llizarov introduced the method of bone transport in a large gap (Figure 1). For bone transport the orthopaedic surgeon can now resect the entire avascular bone and create a large gap. Resection followed by bone transport has revolutionized the better management of chronic Osteomyelitis in children and adult. The Ilizarov method simultaneously addresses the deformity, limb length discrepancy and joint mobility (Figure 2).

\section{Surgical procedure}

Preoperative planning and assessment is very important in treating the chronic osteomyelitis in children. 1. We must palpate arteria dorsalis pedis and posterior tibial artery. 2. Local temperature and color of the foot must be seen. 3 . We must assess with preoperative pulse- Occimeter. If the Ilizarov fixator is stable then the Corticotomy is performed at the proximal metaphyseal or distal metaphyseal region. To eliminate infection vascularization of the osteomyelitic centre is increased by the biological stimulation of corticotomy. Professor Ilizarov said - infection burns in the fire of regeneration and that is absolutely true in case of children osteomyelitis. The gap is closed by gradual controlled coordinated stretching by transporting the segment. $1 \mathrm{~mm}$ per day till the distal end locks the proximal end of the distal fragment. Then the two fragments are compressed together. If the gap is too large, we should introduce a guide wire in between the fragments to keep the bone in anatomical position and to prevent any kind of angulation and rotation which can cause malunion and deformity of the limb. The Ilizarov fixator is kept till the new corticotomy site the fragments are distracted till the limb length is restored. ${ }^{8-10}$

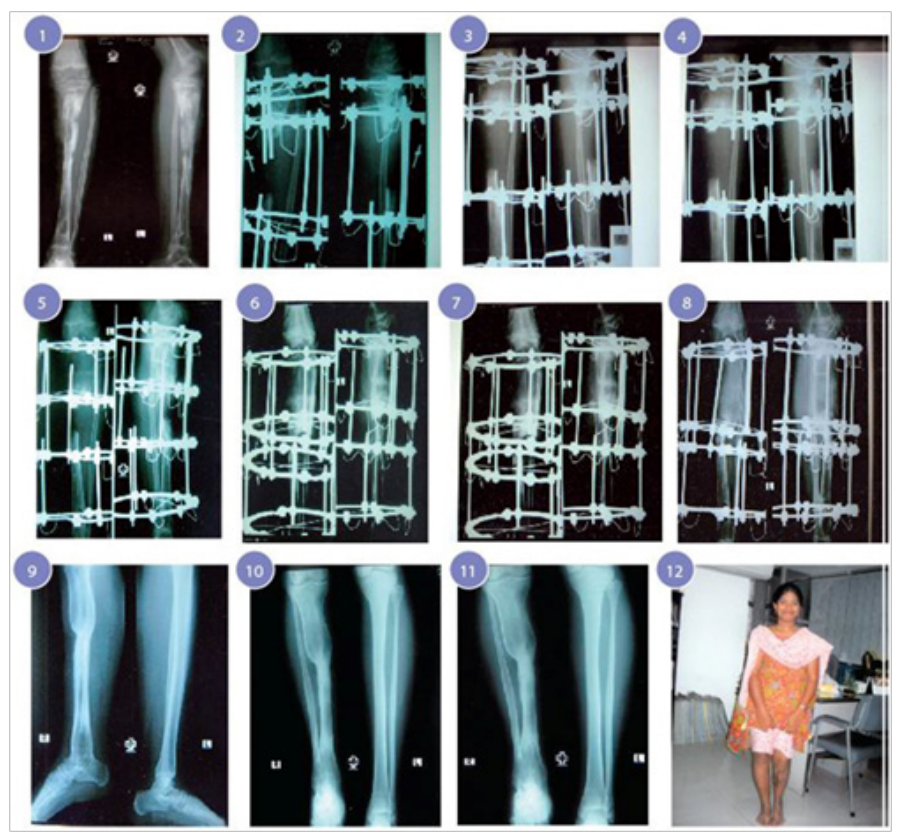

Figure I

Case I I. Pandiaphyseal osteomyelitis of left tibia.

2. Excision of avascular dead bone to create gap non-union 2 nd post OP.

3. Corticotomy \& lengthening performed after 10 days follow up.

4. After 3 weeks follow up.

5.After I month follow up.

6.After I and half months follow up.

7.After 2 months follow up.

8. After 2 and half months follow up.

9, 10, II. Radiographic result after 3 months follow up.

12. Clinical appearance of the patient after 6 months.

\section{Nutritional status}

In Bangladesh, poor nutrition is also a major factor. Complications are a fact of life that every orthopaedic surgeon has to face. Poor nutrition in orthopaedic surgery can make its complications more obvious. We can decrease complication by maintaining appropriate patient nutritional status. ${ }^{7}$

\section{Antibiotics}

First generation cephalosporin are appropriate for prophylactic uses: these drugs are more affective against gram positive cocci. Ciprofloxacin is bactericidal against most gram negative aerobic organisms, including P. Peruginose. ${ }^{7}$ 


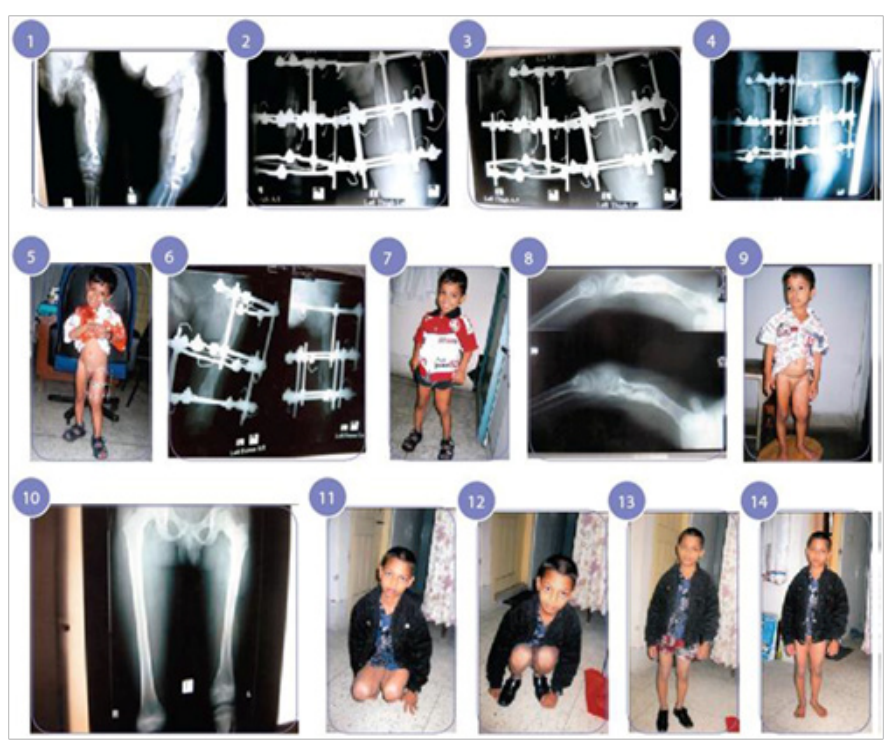

Figure 2

Case 2 I. Pandiaphyseal osteomyelitis with pathological fracture of left femur. 2.5 days after application of Ilizarov.

3. 2 months after application of llizarov.

4. 3 months after application of llizarov.

5. Smiling baby with llizarov in situ.

6. 4 months follow up after application of llizarov.

7. Patient is happy.

8. Radiograph result after 5 months.

9. Clinical appearance of the patient after 5 months.

10. Radiograph after 6 years follow up.

II. Baby can squat.

12. Full range of movements.

13. Clinical appearance of the patient after 6 years.

14. After 6 years of follow up.

\section{Discussion}

Ilizarov technique serves four purposes:

1. To create a mechanical condition necessary for the development of distraction \& compression.
2. To store the new bone forming cells developed during lengthening and deposited along the line of stress and tension.

3. Increase blood circulation for increased metabolic transformation of local tissue.

4. Most importantly the medullary and the periosteal blood supply is not disturbed.

\section{Conclusion}

Chronic osteomyelitis in children with pandiaphyseal osteomyelitis with pathological fracture, including discharging sinus can be treated by stable fixation of Ilizarov apparatus. Sometime radical resection can also be done to create a gap which can be filled up by bone transportation with excellent results.

\section{References}

1. Bari MM. A color atlas of limb lengthening, surgical reconstruction and deformity correction by Ilizarov technique. 2013;pp. 336-339.

2. Bari MM. Correction of Leg Deformities and Restoration of Function of Leg Bones by Ilizarov Technique A color atlas of limb lengthening, surgical reconstruction and deformity correction by Ilizarov technique. 2014;p. 35-121.

3. Cierny G, Zorn KE. Segmental tibial defects. Comparing conventional and Ilizarov methodologies. Clin Orthop Relat Res. 1994;301:118-123.

4. de Pablos J, Barrios C, Alfaro C, et al. Large experimental segmental bone defects treated by bone transportation with mono lateral external distracters. Clin Orthop Relat Res. 1994;298:259-265.

5. Golyakhovsky V, Frankel VH. Ilizarov bone transport in large bone and in severe osteomyelitis. Bull Hosp Jt Dis Orthop Inst. 1991;51(1): 63-73.

6. Green SA, Jackson JM, Wall DM, et al. Management of segmental defects by the Ilizarov intercalary bone transport method. Clin Orthop Relat Res. 1992;280:136-142.

7. Green SA. Osteomyelitis. The Ilizarov perspective. Orthop Clin North Am. 1991;22(3):515-521.

8. Ilizarov GA. The treatment of Pseudarthrosis complicated by Osteomyelitis and the elevation of purulent cavities: Transosseous osteomyelitis. Springer-Verlag, New York, USA, 1992;pp.495.

9. Luca Lazzarini, Mader JT, Calhoun JH. Current concepts review: Osteomyelitis in long bones. J Bone Joint Surg Am. 200486(10):23052318.

10. Simpson AH, Deakin M, Latham JM. Chronic osteomyelitis; the effect of the extent of surgical resection on infection free survival. J Bone Joint Surg Br. 2001;83(3):403-407. 